\title{
Analysis of Voltage Rise Effect on Distribution Network with Distributed Generation
}

\author{
M. A. Mahmud, ${ }^{*}$ M. J. Hossain,${ }^{*}$ H. R. Pota ${ }^{*}$ \\ * The University of New South Wales at the Australian Defence Force \\ Academy, Northcott Drive, Canberra, ACT 2600, Australia (e-mail: \\ Md.Mahmud@student.adfa.edu.au,m.hossain@adfa.edu.au, and \\ H.Pota@adfa.edu.au).
}

\begin{abstract}
Connections of distributed generation (DG) in distribution networks are increasing. These connections of distributed generation cause voltage rise in the distribution network. This paper presents a detail analysis of how does voltage rise on distribution network due to the penetration of distributed generation. This paper also presents how does the voltage rise affect the connection of distributed generation on distribution network. The analysis is done on a simple two-bus distribution network and a radial distribution network with and without DG by using worst case scenario (minimum load maximum generation). Finally, some recommendations are given to counteract the voltage rise effect.
\end{abstract}

Keywords: Distributed generation, distribution network, penetration, voltage rise, worst case scenario.

\section{INTRODUCTION}

Distributed generation is not a new concept but it is an emerging approach for providing electric power in the heart of the power system. In the early age of electricity generation, the distributed generation was the rule, not the exceptions as the first power plants only supplied electricity to customers who are very close to the generating stations. In the last decade, there is a renewed interest for DG due to the technological innovations and change in economic and regulatory environment.

The present status of distributed generation is still at an early stage of development due to the several regulatory, economic, and technical barriers to the integration of DG in electric power systems; see Alderfer et al. (2000). However, there is an ongoing international effort on investigating the necessary arrangements such as an effective market mechanism, and technical and regulatory guidance to provide a sufficient ground for promoting a greater uptake of distributed generation investment. Several authors discuss the potential of DG to defer investments based on its proximity to load demands; see Willis and Scott (2000); Welch (2000); Wright and Formby (2000).

Since the modern distribution systems are designed to accept bulk power from the transmission network and to distribute it to customers, the flow of both real and reactive power is always from the higher to lower voltage levels. However, with significant penetration of distributed generation, the power flows may become reversed and the distribution network is no longer a passive circuit supplying loads but an active system with power flows and voltages determined by the generation as well as load. Hence, there is dramatic changes in the nature of distri- bution networks with distributed generation. A number of studies have built in; see Chiradeja and Ramakumar (2004); Ochoa et al. (2006); Ochoa (2008); Wang and Nehrir (2004); Acharya et al. (2006); Masters (2002), for DG planning models which consider the various technical requirements such as thermal ratings of the equipments, voltage rise, systems fault levels, line drop compensation, reverse power flow capability of the tap-changers, power losses, power quality (such as flicker, harmonics), protection, etc.

Connections of distributed generation in distribution systems are susceptible to voltage rise. Moreover, the impact of losing a single or a few distributed generation following a remote fault may not be significant issue, but the connection or disconnection of a large penetration of distributed generation may become problematic which may lead to sudden appearance of hidden loads and affect the voltage profile of low voltage distribution network. To overcome these problems, the distribution network capacity for distributed generation by using optimal power flow with voltage step constraints is determined in; see Dent et al. (2010). The concepts of voltage steps and voltage rise are related to each other but they are different which is also described in; see Dent et al. (2010). The voltage rise issues due to the distributed generation are discussed nicely through some theoretical background in; see Masters (2002). But the detailed analysis of voltage rise effects is not discussed in; see: Masters (2002). Also there is no indication in; see Masters (2002), about how much DG can be connected to the distribution networks.

The aim of this paper is to present a detailed analysis of voltage rise effect on distribution networks due to DG through a mathematical analysis. This paper also 


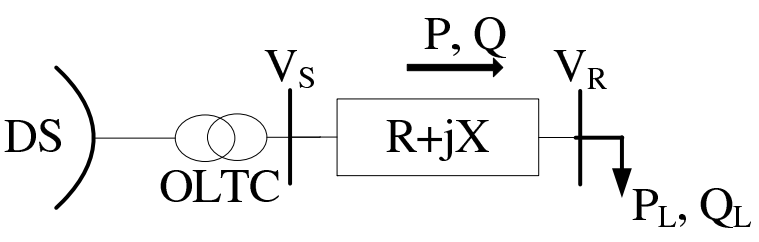

Fig. 1. Conventional Two-bus Distribution System

presents the distribution network capacity for distributed generation in light of voltage rise. The analysis are done on a two-bus distribution system by using worst case scenario. The relation between voltage level, voltage rise and connection cost of distributed generation along with some recommendations to mitigate the voltage rise effects on distribution network are also presented in this paper.

The rest of the paper is organized as follows. An analysis of voltage profile on a two-bus distribution system without distributed generation is presented in Section II. Section III presents an overview of voltage rise on distribution network with distributed generation. In Section IV the worst case scenario of the distribution network with DG is discussed to show how the voltage rise affects the connection of DG on distribution system. The relation between voltage level, voltage rise and connection cost of distributed generation is presented in Section V. The mitigation of voltage rise from a mathematical point of view is discussed in Section VI. Finally, the paper is concluded by brief remarks in Section VII.

\section{VOLTAGE PROFILE OF CONVENTIONAL DISTRIBUTION NETWORK}

Most of the distribution networks are modeled as passive networks with radial configuration and as mentioned in the literature, the flow of power both real $(P)$ and reactive $(Q)$, is always from the higher to lower voltage levels. Since the reactance to resistance ratio $\left(\frac{X}{R}\right)$, for transmission network is $\geq 10$ and that of for distribution network is $\leq 0.5$, therefore the value of resistances in the distribution networks are high. These high resistances lead to the voltage drop along the line from the primary substation to the point of connection of the customer. The amount of voltage drop can be calculated from the analysis of two-bus distribution system as shown in Fig. 1.

In Fig. 1, DS and $O L T C$ stand for the distribution systems and on-load tap-changer respectively, $V_{S}$ is the sending end voltage, $V_{R}$ is the receiving end voltage, $P$ and $Q$ are the real and reactive power flowing through the distribution network to the customer, i.e., these are supplied from distribution substation (DS), and $P_{L}$ and $Q_{L}$ are the real and reactive power of the load. The voltage at the sending end can be written as

$$
\widehat{V}_{S}=\widehat{V}_{R}+\widehat{I}(R+j X)
$$

where $\widehat{I}(I=|\widehat{I}|)$ is the phasor representation of the current flowing through the line. The power supplied from the distribution system can be written as

$$
P+j Q=\widehat{V}_{S} \widehat{I}^{*}
$$

Therefore, the current flowing through the line can be written as

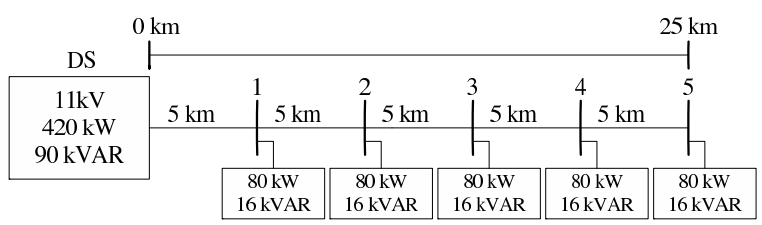

Fig. 2. Conventional Radial Distribution System

$$
\widehat{I}=\frac{P-j Q}{\widehat{V}_{S}}
$$

By using the value of $\widehat{I}$, the sending end voltage can be expressed as

$$
\begin{aligned}
\widehat{V}_{S} & =\widehat{V}_{R}+\frac{P-j Q}{\widehat{V}_{S}}(R+j X) \\
& =\widehat{V}_{R}+\frac{R P+X Q}{\widehat{V}_{S}}+j \frac{X P-R Q}{\widehat{V}_{S}}
\end{aligned}
$$

Therefore, the voltage drop between the sending end and recieving end can be written as

$$
\Delta \widehat{V}=\widehat{V}_{S}-\widehat{V}_{R}=\frac{R P+X Q}{\widehat{V}_{S}}+j \frac{X P-R Q}{\widehat{V}_{S}}
$$

Since the angle between the sending end voltage and the receiving end voltage is very small, the voltage drop is approximately equal to the real part of the drop ? and if the sending end bus is considered as reference bus, the angle of this voltage is 0 , i.e., $\widehat{V_{S}}=\left|V_{S}\right|=V_{S}$. Therefore, the above equation can be approximated as

$$
\Delta V \approx \frac{R P+X Q}{V_{S}}
$$

If the sending end voltage of the system as shown in Fig. 1 is considered as the base voltage, then $V_{S}$ can be assumed as unity. Therefore, equation (1) can be written as follows:

$$
\Delta V \approx R P+X Q
$$

The voltage level at each connection point of the load is very important for the quality of the supply. Since there are no internationally agreed rules that define allowed steady-state voltage range, the maximum permitted voltage variation on each bus-bar is defined by some technical regulations and or specific contracts. The voltage level at each point of load can easily be described by using the radial distribution network as shown in Fig. 2.

In Fig. 2, an $11 \mathrm{kV}, 420 \mathrm{~kW}, 90 \mathrm{kVAR}$ distribution is supplied power to a total load of $400 \mathrm{~kW}, 80 \mathrm{kVAR}$. Here, the total length of the radial distributor is $25 \mathrm{~km}$ and $80 \mathrm{~kW}, 16 \mathrm{kVAR}$ load is connected after each $5 \mathrm{~km}$. The resistance of the line is $0.625 \mathrm{ohm} / \mathrm{km}$ and the reactance is $0.3125 \mathrm{ohm} / \mathrm{km}$. The voltage profile of this radial distribution system is shown in Fig. 3.

In Fig. 3, the two dashed lines specify the range of the allowable voltage variation along the distribution system. In most cases, the allowable voltage variation along the distribution network is $\pm 6 \%$, but it may vary depending on the regulations of each country. In our case, we have considered the allowable voltage variation as $\pm 6 \%$. From 


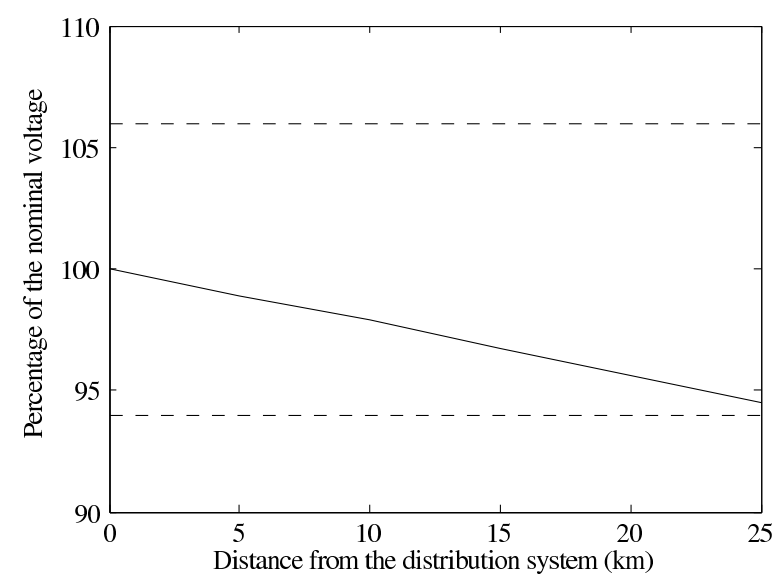

Fig. 3. Voltage Profile (The dashed lines represent the specified $\pm 6 \%$ voltage limit and the solid line represents the percentage drop in voltage through the system)

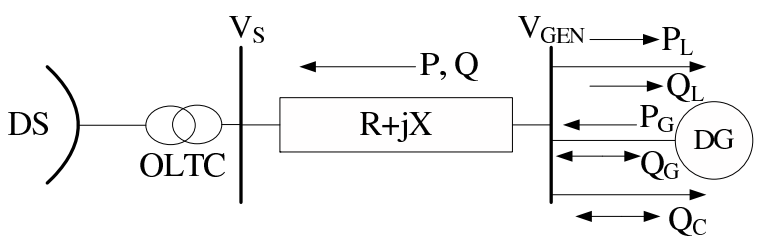

Fig. 4. Two-bus Distribution System with DG

Fig. 3, it is seen that the voltage variations are within the specified limit of $\pm 6 \%$. If the line is more longer or the loads are greater, the farthest nodes of the network falls outside the permitted voltage range. In this case, the electricity distribution network service providers (DNSPs) run their networks within the allowable voltage ranges as defined by the regulation through the use of automatic voltage controller (AVC), on-load tap-changers and shunt compensators.

\section{VOLTAGE RISE ON DISTRIBUTION NETWORK WITH DISTRIBUTED GENERATION}

The voltage profile in the conventional distribution system is stable. When generators are connected to the distribution system, the power flow and the voltage profiles are affected as well as the system is no longer passive but active. In order to export power, a generator is likely to have to operate at a higher voltage as compared to the other nodes where it is supplied power. This can be explained by using equation (2). In this case, the receiving end voltage $\left(V_{R}\right)$ will be

$$
V_{R} \approx V_{S}+R P+X Q
$$

as the direction of the power flow is reversed. Thus, the voltage at the point of connection of the generator will rise above the sending end voltage which can be more clarified through Fig. 4.

In Fig. 4, a distributed generator (DG) is connected where the voltage $\left(V_{G E N}\right)$ is assumed to be $11 \mathrm{kV}, P_{G}$ and $Q_{G}$ are the generated active and reactive power respectively by the DG, $P_{L}$ and $Q_{L}$ are the active and reactive power of the load respectively and $Q_{C}$ is reactive power of the

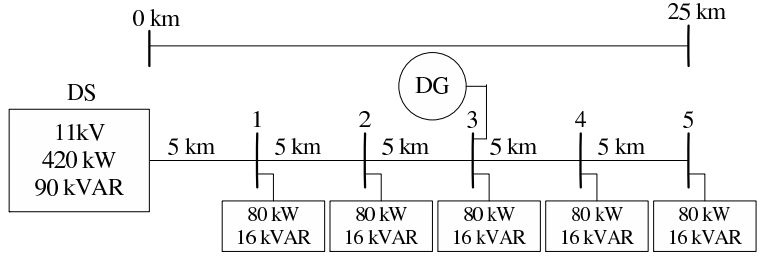

Fig. 5. Radial Distribution System with DG

shunt compensator. This DG altogether with load and compensator is connected to the distribution system (DS) via overhead distribution line with impedance $R+j X$ and through OLTC. The voltage rise along the distribution network as shown in Fig. 4 can be written as follows:

$$
\Delta V=V_{G E N}-V_{S} \approx \frac{R P+X Q}{V_{G E N}}
$$

where, $P=\left(P_{G}-P_{L}\right), Q=\left( \pm Q_{C}-Q_{L} \pm Q_{G}\right)$. If $V_{G E N}$ is expressed in terms of per unit, then equation (4) can be written as

$$
\Delta V=V_{G E N}-V_{S} \approx R\left(P_{G}-P_{L}\right)+X\left( \pm Q_{C}-Q_{L} \pm Q_{G}\right)
$$

The generators always export active power $\left(+P_{G}\right)$ and may export or import reactive power $\left( \pm Q_{G}\right)$, whereas the load consumes both active $\left(-P_{L}\right)$ and reactive $\left(-Q_{L}\right)$ power and the compensators may export or absorb only reactive power $\left( \pm Q_{C}\right)$. Recently, small synchronous generators though combined heat power (CHP) generation scheme, small wind turbine, photovoltaic (PV) are widely used as distributed generators. In CHP generation scheme, the synchronous generator exports real power when the electrical load of the systems falls below the output of the generator but it may absorb or export reactive power depending on the setting of the excitation system of the generator. The wind turbine also exports real power but it absorbs reactive power as its induction generator requires a source of reactive power to operate. The photovoltaic (PV) systems are used to export of real power at a set power factor but may introduce harmonic currents. Therefore, the power flows through the circuits may be in either direction depending on the relative magnitudes of the real and reactive network loads compared to the generator outputs and any losses in the network. This can be demonstrated by connecting distributed generator as shown in Fig 5 to the conventional radial distribution system which is shown in Fig. 2.

In Fig. 5, a distributed generator is connected $15 \mathrm{~km}$ away from the primary distribution system (DS). To demonstrate the effect of voltage rise, we have considered the following two cases:

- A $240 K W$ generator, operating at unity power factor is considered as distributed generator. The output of this generator is equal to the downstream demand, so the direction of the power flow is not altered. At this stage, the voltage profile of the system is shown in Fig. 6. The nominal voltage of DS is controlled at $100 \%$. From Fig. 6, it is seen that the voltage along the system drops in some parts and rises in some other 


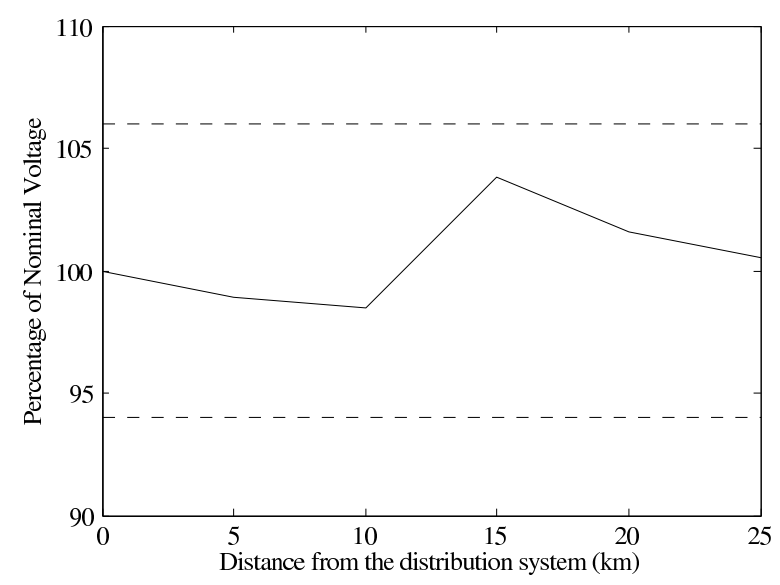

Fig. 6. Voltage Profile of the DS with $240 \mathrm{KW}$ DG (The dashed lines represent the specified $\pm 6 \%$ voltage limit and the solid line represents the percentage drop in voltage through the system)

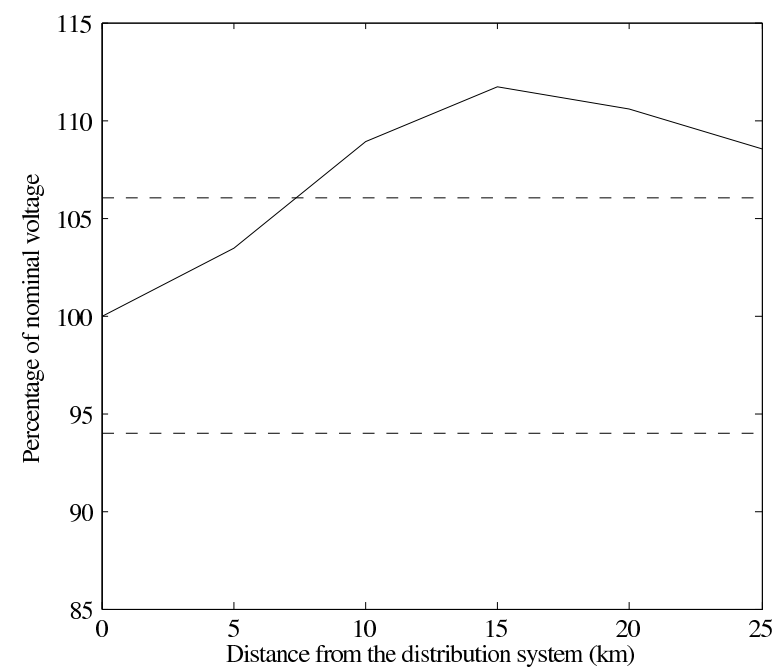

Fig. 7. Voltage Profile of the DS with $1 \mathrm{MW} \mathrm{DG}$ (The dashed lines represent the specified $\pm 6 \%$ voltage limit and the solid line represents the percentage drop in voltage through the system)

parts. But the variation is within the specified limits.

- In this case, $240 K W$ generator is replaced by a $1 M W$ generator. This increased amount of generation reverses the power flow through the line, from the generator towards the DS. The voltage profile of DS with $1 M W$ of distributed generation is shown in Fig. 7. From Fig. 7, it is seen that the voltage in some parts of the system rises above the permitted $+6 \%$ voltage limit.

Due to the large penetration of DG into the DS, the voltage at the DS rise considerably and the flow of power is in both direction. Therefore, before connecting a generator to the distribution system, a DNSPs must consider whether the power may be exported back or not to the main grid and also must ensure that the transformer's tap-changers are capable to operate with reverse power flow.

\section{DISTRIBUTION NETWORK WITH WORST CASE SCENARIO}

The distributed generators are connected to the distribution system due to the technological innovations and change in economic and regulatory environment as well as to meet the increased load demand. From equation (5), we can write,

$$
P_{G} \approx \frac{V_{G E N}-V_{S}+R P_{L}-X\left( \pm Q_{C}-Q_{L} \pm Q_{G}\right)}{R}
$$

From equation (6), it is clear that the level of generation that can be connected to the distribution system depends on the following factors:

- voltage at the primary DS

- voltage level of the receiving end

- size of the conductors as well distance from the the primary DS

- load demand on the system

- other generation on the system

When a generator is to be connected to the distribution system, the DNSP should consider the worst case operating scenarios to easily demonstrate the relationship between voltage rise and the DG connected to the DS and also they should ensure that their network and customer will not be adversely affected. Generally, these worst case scenarios are:

- minimum load maximum generation

- maximum load minimum generation

- maximum load maximum generation

The amount of voltage rise on a distribution system can easily be described through worst case scenario. This can be done by using the simple algebraic equation (5). If we consider the worst case scenario as minimum load and maximum generation, then we can write:

$$
P_{L}=0, \quad Q_{L}=0, \quad \text { and } \quad P_{G}=P_{G \max }
$$

Again, if we assume that the system is operating at unity power factor, then $\pm Q_{G}$ and $\pm Q_{C}$ will be zero. In this condition equation (5) can be written as:

$$
\Delta V_{\text {worst }}=V_{G E N \max }-V_{S} \approx R P_{G \max }
$$

From equation (7), it is clear that the voltage rise depends on the resistance of the distribution line and the power supplied by the distributed generator. If the resistance of the distribution line is constant, then we can write

$$
\Delta V_{\text {worst }} \propto P_{G \max }
$$

Therefore, the voltage in distribution network with distributed generation is directly proportional to the amount the active power supplied by the distributed generators.

There is a linear relationship between the voltage rise and the amount of active power supplied by distributed generators. Therefore, the voltage rise is more onerous when there there is no demand on the system, as all the generation is exported back to the primary distribution system. The voltage rise in the distribution system also 
limits how much distributed generation can be connected. This can be shown by the simple algebraic equation (7).

From equation (6), we can write

$$
P_{\text {Gmax }} \approx \frac{V_{G E N \max }-V_{S}}{R}
$$

The capacity of the generator that can be accommodated in the existing system is clearly limited by the maximum voltage at the distributed generator connected busbar which can be written as

$$
P_{G \max } \leq \frac{V_{G E N \max }-V_{S}}{R}
$$

Therefore, from the worst case scenario it is seen that the resistance of the line as well as the voltage rise along the system is critical for the amount of generation that can be connected.

\section{VOLTAGE LEVEL, VOLTAGE RISE, AND CONNECTION COST}

From the distributed generation perspective, one of the most important questions related to the policy of connecting distributed generation is the voltage level to which generation should/could be connected, as this has major a impact on the overall profitability of generation projects.

The overall connection costs may considerably alter the cost base of a distributed generator and are primarily driven by the voltage level to which the generator is connected. Generally, but not exclusively, the higher the voltage level the greater the connection cost. In order to secure the viability of a generation project, developers and operators of distributed generation would prefer to be connected at the lowest possible voltage level. On the other hand the higher the connecting voltage the lower the impact that distributed generation has on the performance of the local network, particularly in terms of steady state voltage profile and power quality. Therefore, the network operators generally prefer connecting distributed generation to higher voltage levels. These two conflicting objectives need to be balanced appropriately, and may require an in-depth technical and economic analysis of the alternative connection designs and the ability to manage operation of the network including the dispatch of generation and control of network facilities such as OLTCs and other reactive compensation devices. In this respect, the voltage rise effect which a generator connected to a weak network could produce is particularly critical.

\section{MITIGATION OF VOLTAGE RISE}

The large penetration of distributed generation on DS causes an excessive voltage rise. Traditionally, DS is equipped with over voltage protection relay to protect it during the over voltage. The over voltage protection scheme may permanently disconnect the distributed generators or even it may disconnect the DS from the main grid which may cause serious economical damage for the customers as well as DNSPs. The voltage rise on DS can be mitigated through the following approaches:

- Resistance reduction approach

- Reactive power compensation approach
- Coordinated voltage control approach

- Generation curtailment approach

The above mentioned issues are discussed in details in the following subsections:

\subsection{Resistance reduction approach}

If the amount of the connected DG to a DS system is constant, then from equation (9) we can write

$$
\Delta V_{\text {worst }} \propto R
$$

From equation (11), it is seen that the worst case voltage rise which consider maximum DG penetration, is directly proportional to the the resistance of the line. Therefore, if the resistance of the line is reduced, the voltage rise on the DS will also be be reduced. The resistance of a line can be reduced by increasing the conductor size. But it is very difficult to change all the existing network infrastructure to reduce the line resistance. Therefore, it can be proposed that before constructing a new DS, the DNSPs should consider the reduced value of line resistance to make provisions for large DG.

\subsection{Reactive power compensation approach}

From equation (5), we can write

$$
\Delta V=V_{G E N}-V_{S} \approx R\left(P_{G}-P_{L}\right)+X Q_{\text {import }}
$$

where, $Q_{\text {import }}= \pm Q_{C}-Q_{L} \pm Q_{G}$. If we consider the worst case scenario and the operation of the distributed generators at other than unity power factor, equation (12) can be re-written as

$$
\Delta V_{\text {worst }} \approx R P_{G \max }+X Q_{\text {import }}
$$

From equation (12), it is seen that if the amount of imported reactive power is increased, i.e., with higher negative values of $X Q_{\text {import }}$, the voltage rise on the distribution system will be reduced. The amount of reactive power that can be imported generally depends on the parameters of the generators. Typically, a synchronous generator can import power at a 0.95 power factor whereas a wind turbine with uncompensated induction generator can import power at around a 0.9 power factor.

It is also important to bear in mind that absorbing reactive power would lead to an increase in losses and the loading on circuits. The transient voltage rise may occur in DS duo to the effects of generator tripping. It may take the transformer tap-changers at the primary DS several seconds to respond and restore the voltages. Under this circumstances, DNSPs should use a switched capacitor bank or other form of reactive power compensation to restore the system voltages.

\subsection{Coordinated voltage control approach}

In conventional passive distribution network, it is common practice for DNSPs to maintain the primary DS above the nominal voltage to ensure that the system voltages remain within the specified $-6 \%$ voltage limit as the voltage drops 
like Fig. 3. But when distributed generators are connected, the scenarios change like Fig. 7.

As we know,

$$
\Delta V_{\text {worst }}=V_{G E N \max }-V_{S}
$$

From equation (14), it is seen that by regulating the sending end voltage, i.e. primary DS voltage, we can change the voltage drop. This can easily be done by using OLTC connected to the system in short distribution system but in case of long distribution system which includes many distribution transformers it may be impractical. However, in more complex network, the value of this voltage, and the corresponding tap position of the OLTC, would have to be optimized.

\subsection{Generation curtailment approach}

It is important to observe that the probability of such extreme situation (coincidence of minimum load with maximum generation) actually occurring is generally low, and hence it may be beneficial to accommodate a larger distributed generator and curtail it when voltage at busbar where it is connected, rises to that of the limit. The effect of generation curtailment on the capacity that can be connected is given by the equation below.

$$
P_{\text {Gmax }} \approx P_{\text {Gcur }}+\frac{V_{G E N \max }-V_{1}}{R}
$$

Equation (15) can be re-written as

$$
\Delta V_{\text {worst }} \approx R P_{\text {Gmax }}-R P_{G c u r}
$$

From equation (16) it is seen that voltage rise can be reduced through generation curtailment. The likelihood of the coincidence of minimum load with maximum generation will determine the total annual energy curtailed. As the price of electricity is primarily driven by load demand, and generation curtailment occurs typically during periods of low load, the value of this energy curtailed is likely to be relatively low.

\section{CONCLUSION}

The voltage rise scenarios on a distribution a distribution network under different circumstances are analyzed. From the analysis it is shown that there is no serious voltage rise problem on conventional distribution network and distribution network with small amount of DG. When penetration of DG is increased, there is onerous voltage rise effect on the system which might cause economical damage. This voltage rise effect on the distribution network is described through the worst case scenario. The worst case scenario also estimates the amount of the maximum distributed generation that can be connected to the distribution system. The voltage level, voltage rise, and connection costs are related to each other. Mitigations of voltage rises are analyzed through resistance reduction approach, reactive power compensation approach, coordinated voltage control approach and generation curtailment approach. Future works will deal with the dynamic analysis of this distribution system with different types of distributed energy resources such as CHP, wind generators, PV etc.

\section{REFERENCES}

Acharya, N., Mahat, P., and Mithulananthan, N. (2006). Analytical approaches for optimal placement of distributed generation sources in power systems. Int. J. Elect. Power and Energy Syst., 28(10), 669-678.

Alderfer, B., Eldridge, M., and Starrs, T. (2000). Making connection: Case studies of interconnection barriers and their impact on distributed power projects. National Renewable Energy Laboratory.

Chiradeja, P. and Ramakumar, R. (2004). An approach to quantify the technical benefits of distributed generation. IEEE Trans. on Energy Conversion, 19(4), 764-773.

Dent, C.J., Ochoa, L.F., and Harrison, G.P. (2010). Network distribution capacity analysis using OPF with voltage step constraints. IEEE Trans. on Power Systems, 25(1), 296-304.

Masters, C.L. (2002). Voltage rise: the big issue when connecting embedded generation to long $11 \mathrm{kV}$ overhead lines. IET Power Engineering Journal, 16(2), 5-12.

Ochoa, L.F. (2008). Time-series based maximization of distributed wind power generation integration. IEEE Trans. on Energy Conversion, 23(3), 968-974.

Ochoa, L.F., Padilha-Feltrin, A., and Harrison, G.P. (2006). Evaluating distributed generation impacts with a multiobjective index. IEEE Trans. on Power Delivery, 21(3), 1452-1458.

Wang, C. and Nehrir, M.H. (2004). Analytical approaches for optimal placement of distributed generation sources in power systems. IEEE Trans. on Power Systems, 19(4), 2068-2076.

Welch, G. (2000). Distributed generation planning. In Proc. of IEEE Summer Meeting.

Willis, H.L. and Scott, W.G. (2000). Distributed Power eneration: Planning and Evaluation. Marcel Dekker, New York.

Wright, A.J. and Formby, J.R. (2000). Overcoming barriers to scheduling embedded generation to support distribution networks. EA Technology-Department of Trade and Industry. 